compared with transpyloric feeding. Arch Dis Child 1984;59:131-5.

${ }^{2}$ Eyal F, Sagi E, Arad I, Auital A. Necrotising enterocolitis in the very low birthweight infant: expressed breast milk feeding compared with parenteral feeding. Arch Dis Child 1982;57: 274-6.

3 Yu UYH, James B, Hendry P, MacMahon RA. Total parenteral nutrition in very low birthweight infants: a controlled trial. Arch Dis Child 1979;54:653-61.

\section{Oral rehydration fluids}

Sir,

Tripp and Candy have reviewed the present state of oral rehydration clearly and concisely. ${ }^{1}$ I would like to make a few comments.

In most places where the World Health Organisation (WHO) oral rehydration solution is used a large proportion of patients will have varying degrees of malnutrition. Thus, experience of treatment in developing countries is not necessarily applicable to developed countries where intravenous fluids are available. In developing countries most children who die from dehydration die because they have not received intravenous fluids appropriately, or at all. The purpose of oral rehydration solution is to prevent them getting to this stage. Although WHO oral rehydration solution can be used successfully, even in severely dehydrated children if carefully managed, it does not negate the necessity to have facilities for intravenous treatment. About 10 to $15 \%$ of children in hospital treated with oral rehydration solution will need the addition of intravenous fluids to achieve rehydration. ${ }^{2}$ Unfortunately many children with moderate to severe dehydration die when given oral rehydration solution because it is given unsupervised resulting in progression of dehydration, and intravenous fluids are not given or are given too late.

In developed countries most children with diarrhoea who are not dehydrated or only mildly so are adequately treated with a low osmolar solution, and those more severely dehydrated with intravenous fluids. A $100 \%$ cure rate is the aim. I do not see why, unless the health facilities deteriorate in these countries, the practice should be changed. In fact rehydrating a moderately dehydrated child with oral rehydration solution is much more time consuming for nursing staff than administration of intravenous fluids with the aid of an infusion pump.

Oedema in children given oral rehydration solution may be a symptom of malnutrition rather than of overhydration. Many malnourished children develop oedema for the first time after rehydration with the WHO oral rehydration solution. This does not necessarily mean that they are adequately rehydrated but more that the sodium load of oral rehydration solution is too much for their already compromised state, which includes a reduced plasma oncotic pressure and impaired renal tubular function.

It is suggested that the potassium concentration of the WHO oral rehydration solution is too low to replace the total body potassium deficit that is commonly found in malnourished children with diarrhoea. What is more important than increasing the potassium concentration of the present solution, which ideally is only used for rehydration and not maintenance, is to give potassium supplements for some days or weeks after rehydration in the form of potassium salts, bananas, coconut water, or citrus fluids. In the case of the occasional child who presents with symptoms of hypokalaemia such as hypotonia, abdominal distention, and ileus only high doses of intravenous potassium followed by an oral potassium for two to three weeks will suffice.

Regarding appropriate technology, it is important to separate the children with nil to mild dehydration who can be adequately treated with a variety of fluids from the moderate to severely dehydrated child who in the absence of intravenous treatment can only be rehydrated by a carefully managed high osmolar solution. The osmolality of 'home based solutions' can vary enormously. A low osmolar solution will not be adequate for the treatment of these children.

Lastly, it has been stated that neonates can be successfully rehydrated by the WHO oral rehydration solution. The physiology of the neonate in developing countries is no different to that in developed countries where solutions containing $0 \cdot 18 \mathrm{~N}$ saline are used for rehydrating neonates. At present, in view of the neonates' limited ability to excrete sodium, it would seem unwise to advocate the universal use of WHO oral rehydration solution for management of dehydration in neonates until it has been shown that it is safe when given unsupervised. Unfortunately, with the number of dehydrated infants presenting to health centres in developing countries, supervision of rehydration is the exception rather than the rule.

J B S Coulter Liverpool School of Tropical Medicine, Pembroke Place, Liverpool L3 $5 Q A$

Sir,

Drinks will not help a child in circulatory collapse from diarrhoea; liquid by vein will.

Unless enthusiasts for oral rehydration make this clear children may die and an old and valued remedy for moderate or early enteritis will be blamed. Drs Tripp and Candy in their annotation ${ }^{1}$ should surely have reminded us that in the paper by Santosham et al, ${ }^{3}$ on which they rely heavily, all children with severe diarrhoea (shock or $10 \%$ dehydration) received intravenous treatment before entering the trial.

$\mathrm{T}$ H Hughes-Davies 6 Tavistock Court, Tavistock Square, London WC1

Drs Tripp and Candy comment:

One of the reasons for writing our annotation ${ }^{1}$ was to review the controversies and stimulate discussion on the subject of oral rehydration treatment for acute diarrhoea. We were therefore grateful to receive letters from Drs Hughes-Davies and Coulter and for this opportunity to comment. Both correspondents have highlighted the need 